\title{
Barrier formation of micro-crack interface and piezoelectric effect in coal and rock masses
}

\author{
Xiangfeng $\mathrm{Lv}^{\mathrm{a}, \mathrm{b}, *}$, Yishan Pan $^{\mathrm{c}}$, Xiaochun Xiao $^{\mathrm{c}}$, Aiwen Wang ${ }^{\mathrm{c}}$ \\ a Beijing Municipal Engineering Research Institute, Beijing 100037, China \\ ${ }^{\mathrm{b}}$ Institute of Mechanics, Chinese Academy of Sciences, Beijing 100190, China \\ c School of Mechanics and Engineering, Liaoning Technical University, Fuxin 123000, China
}

\section{A R T I C L E I N F O}

Article history:

Received 21 April 2012

Received in revised form 9 April 2013

Accepted 2 August 2013

Available online 10 September 2013

Keywords:

Coal and rock mass

Crack interface and tip

Sliding friction

Interface barrier

Charge

\begin{abstract}
A B S T R A C T
The interface barrier formation mechanism of micro-cracks of coal and rock mass is investigated by using theoretical analysis and piezoelectric experiments. It is found that the micro-cracks of coal or rock expand constantly under external loads, while the slip, dislocations and inhomogeneous deformation of crack interface and lattice cause charge breakthrough, and then, barrier form on the crystal interface. The generation process of charge is accompanied by the friction of micro-crack slip, and the dislocation and deformation are analyzed theoretically. A theoretical model of micro-crack slip friction charge is established. Furthermore, piezoelectric law of coal or rock deformation and fracture is studied experimentally. The results show that cracks expand vertically more seriously than horizontally, and vertical cracks expand first, followed by horizontal ones, which expand continuously till crashed at the end of the coal sample. Crack interface value of potential barrier increases constantly in the compression process of coal sample. And before the coal sample gets crashed, sliding friction between crack interfaces reaches the peak, together with the potential barrier. Moreover, extreme acceleration of transfer speed of charge is based on extreme multiplying potential value of crack interface. Both crack interface barrier and potential amplitude get change dramatically before the coal sample fracture, which will help to get the precursor information of coal sample rupture. More importantly, the monitoring results can predict the extent and state of coal and rock rupture.
\end{abstract}

(c) 2013 Elsevier Ltd. All rights reserved.

\section{Introduction}

The deformation and fracture process of coal or rock is accompanied by the charge generation under external loads. Studying the charge generated by coal or rock deformation and fracture is significantly important for monitoring coal and rock mass deformation and failure [1-3]. Domestic and foreign researchers had carried out a lot of research on the phenomenon of rock fracture charged. And the subjects are mostly limited to marble, granite and other hard rock. Ponomarev conducted investigations on the natural charged changes of rupture of the rock under uniaxial cyclic loading [4]. Brady and Rowell [5] have carried out a laboratory investigation of the electrodynamics of rock fracture. He et al. [6] also studied the electromagnetic emission characteristics in roof failure. The surface charge phenomenon of granite under uniaxial compression is studied by $\mathrm{Wu}$ [7]. It shows that the rock surface positive charge can be generated during loading

\footnotetext{
* Corresponding author at: Institute of Mechanics, Chinese Academy of Sciences, Beijing 100190, China.

E-mail address: lvxiangfeng2006@126.com (X. Lv).
}

process. The potential variation of granite and marble in deformation and fracture process under uniaxial compression is studied by Enomoto [8]. The instantaneous potential of about $40 \mathrm{mV}$ and about $35 \mathrm{pA}$ current signal is obtained in the fast loading test on granite and gabbro by Takeuchi [9]. However, there are few studies on the generation process of charge for coal and rock deformation and fracture [10-13]. Can coal and rock mass deformation and fracture process produce charge? What is the mechanism of charge and what is the charge generation law? The charge generation mechanism and piezoelectric law of coal or rock fracture are not clearly understood. Therefore, study on formation of micro-crack interface barrier and piezoelectric effect of coal and rock mass, which can provide strong guidance for the charge monitoring on coal or rock burst, is important both in theory and in engineering applications.

In this paper, the interface barrier formation mechanism of micro-cracks of coal and rock mass is investigated by using a combination method of theoretical analysis and piezoelectric experiments. The charge accompanying with the friction process of micro-crack slip dislocation and deformation is analyzed theoretically. At the same time, the theoretical model of micro-crack slip friction charge is established. And then, piezoelectric law of 
coal or rock deformation and fracture is studied experimentally. The charge generation mechanism of coal or rock deformation and fracture is identified. The results provide a reliable basis for monitoring on the precursor information of coal and rock mass deformation.

\section{Discussion on barrier formation mechanism of micro-crack interface}

\subsection{Barrier formation mechanism of micro-crack interface}

Coal and rock mass includes a lot of tiny cracks. And microcracks close first under external loads. With the external loads increase continuously, new cracks will emerge and the original cracks will grow in coal-rock body. At the same time, the crack surface and the cutting edge generate dislocations and slip [14], which result in coal or rock volume expansion and deformation. There must be interface friction accompanied by the movement and transfer of atoms in the deformation process. Also, positive and negative charges accumulate on different directions. A vacancy formed in the transfer process. For the same, atoms can also jump to different space because of constantly reduplicative movement and transfer. So a state of equilibrium achieves ultimately. Fig. 1 shows the mechanism diagram of the coal rock micro-crack tip dislocation.

\subsection{Vacancy generated by dislocation}

Newborn cracks, expansion and deformation of existing cracks of coal or rock occur under external loads. Atoms jump from one lattice position to another, which cause vacancy formation at original lattice position. It can be also understood as the atom jumps to the vacancy $[15,16]$ and the vacancy transfers from one grid location to another. Atoms jumping movement result in diffusion generated in the micro-cracks rupture process of coal and rock mass deformation. The interface charge generation of coal-rock is also the atomic jump process. While, only the atom get the jump conditions that the value is more than the barrier, can the atoms jump cross the barrier (the maximum of the potential energy between two gaps). Fig. 2 shows the schematic illustration for the formation of vacancy. Fig. 3 indicates atoms at interstitial sites with the relative minimum potential energy.

\subsection{Calculation of the vacancy formation energy}

Vacancy formation can be envisaged for two steps. First, a positive charge is uniformly distributed in the matrix and remove the uncharged atoms, so that the entire crystal maintains a neutral power and then a positive ion vacancy is formed. Second, atom which resume its positive charge is placed on the surface edge. Therefore, the vacancies [17] forms including two parts of the increase of the electrostatic potential energy and electron kinetic energy.

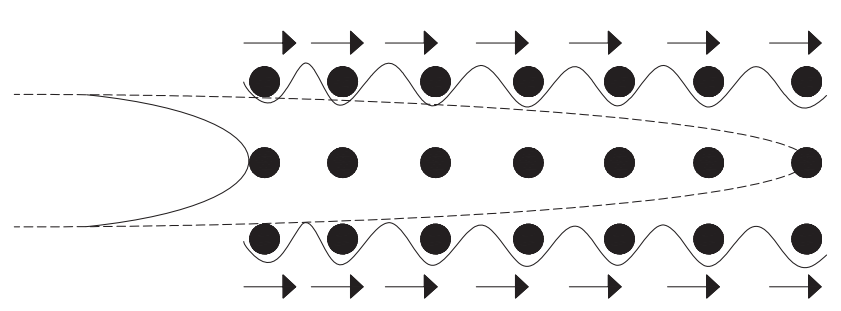

Fig. 1. Micro-crack tip dislocations.

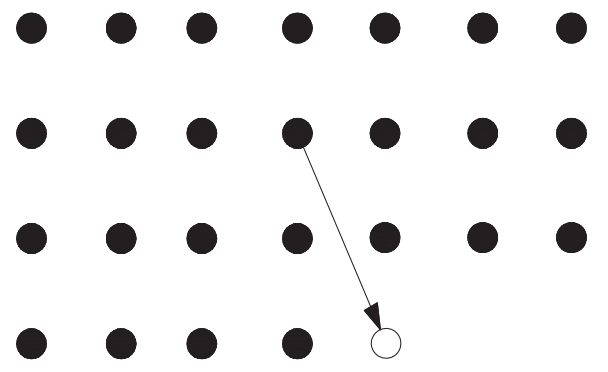

Fig. 2. Schematic of the vacancy formation.

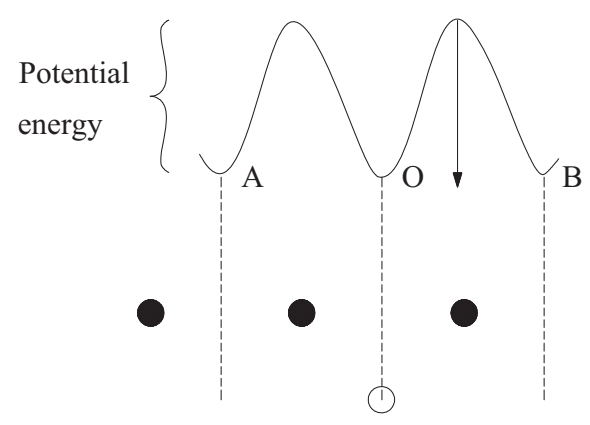

Fig. 3. Atom barrier.

\subsubsection{Increase of the electrostatic potential}

Assume vacancy formed at a certain point $p$ in the crystal. The electrostatic potential in the distance of $r$ is $V_{P}(r)$. The electrostatic potential energy of an electron is $-V_{P}(r) e, n$ represents the electron density; $n=8 \pi / 3 h^{3} p_{0}^{3}$. The electrostatic potential increased by the vacancy formation is:

$E_{1}=-n e \int_{0}^{R} 4 \pi r^{2} V_{P}(r) d r$

where, $R$ is the actual effective range of the charge field formed by the vacancy formation.

If the maximal energy of the electrons is expressed by $-e V_{0}$, and we get:

$-e V_{0}=\frac{p_{0}^{2}(r)}{2 m}-e V_{P}(r)$

where, $p_{0}(r)$ and $V_{P}(r)$ are the electron momentum and potential, respectively. $m$ is the electron mass.

The maximal energy of electrons is equal to the free-density which is $-e V_{0}=E_{F}^{0}$, and substitute it into Eq. (2):

$n(r)=\frac{8 \pi}{3 h^{3}}\left\{2 m e\left[V_{P}(r)+\frac{E_{F}^{0}}{e}\right]\right\}^{3 / 2}$

The distribution of negative charge density at $r$ is

$\rho^{-}=-e n(r)=-\frac{8 \pi}{3 h^{3}}\left\{2 m e\left[V_{P}(r)+\frac{E_{F}^{0}}{e}\right]\right\}^{3 / 2}$

The positive charge density is

$\rho^{+}=e n=e \frac{8 \pi}{3 h^{3}} P_{0}^{3}=e \frac{8 \pi}{3 h^{3}}\left(2 m E_{F}^{0}\right)^{3 / 2}$ 
Charge density at $r$ is

$\rho(r)=\rho^{+}+\rho^{-}=e \frac{8 \pi}{3 h^{3}}\left(2 m E_{F}^{0}\right)^{3 / 2}-e \frac{8 \pi}{3 h^{3}}\left(2 m E_{F}^{0}\right)\left[1+\frac{e V_{P}(r)}{E_{F}^{0}}\right]^{3 / 2}$

Expend $\left[1+e V_{P}(r) / E_{F}^{0}\right]^{3 / 2}$ and get the first two terms, the charge density is formulated as:

$\rho(r)=-\frac{4 \pi}{h^{3}}(2 m)^{3 / 2} e^{2} V_{P}(r)\left(E_{F}^{0}\right)^{1 / 2}$

Unit volume state distribution function for free electrons:

$N(E) d E=4 \pi \frac{(2 m)^{3 / 2}}{h^{3}} E^{1 / 2} d E$

where, $h$ and $m$ are constant. $N(E)=C E^{1 / 2}, C=4 \pi(2 m)^{3 / 2} / h^{3}$. Thus,

$\rho(r)=-e^{2} V_{P}(r) N\left(E_{F}^{0}\right)$

Formula (9) shows the relationship between the electrostatic potential $\left(V_{P}(r)\right)$ and net charge distribution.

Assume that the space point is a point charge. Then,

$e=\int_{0}^{R} 4 \pi r^{2} \rho(r) d r$

Substitute the above equation into the formula (9):

$N\left(E_{F}^{0}\right) \int_{0}^{R} 4 \pi r^{2} e V_{P}(r) d r=-1$

Substituting formula (1):

$E_{1}=\frac{n}{N\left(E_{F}^{0}\right)}$

The electron density can be expressed as:

$n=\int_{0}^{E_{F}^{0}} N(E) d E=\int_{0}^{E_{F}^{0}} C E^{\frac{1}{2}} d E=\frac{2}{3} C\left(E_{F}^{0}\right)^{3 / 2}$

The increment of the electrostatic potential is:

$E_{1}=\frac{2}{3} E_{F}^{0}$

\subsubsection{Reduction of the electron kinetic energy}

Electronic charge density reduces caused by crystal volume expansion. Then we get,

$E_{F}^{0}=\left(\frac{3 n}{2 C}\right)^{2 / 3}=\left(\frac{3 N}{2 V C}\right)^{2 / 3}$

where, $N$ is the number of crystal atoms. $V$ is the crystal volume. $\Delta V$ is the crystal volume expansion. $\Delta E_{F}^{0}$ is the fees density change.

Knowing that $\Delta V=\frac{V}{N}$, on the type points can be:

$\Delta E_{F}^{0}=-\frac{2}{3}\left(E_{F}^{0}\right) \frac{1}{N}$

The original average kinetic energy of each electron is $3 / 5 E_{F}^{0}$. The volume expansion caused by changes in total kinetic energy of the electronic is

$E_{2}=N\left(\frac{3}{5} \Delta E_{F}^{0}\right)=\frac{2}{5} E_{F}^{0}$

Neglecting the effect of other distortion shape, the vacancy formation energy is:

$E_{f}=E_{1}+E_{2}=\frac{2}{3} E_{F}^{0}-\frac{2}{5} E_{F}^{0}=\frac{4}{15} E_{F}^{0}$

\section{Theoretical model of crack sliding friction electricity}

The relative slip and deformation $[17,18]$ which cause crystal volume expansion between the crack tip lattice in the crack propagation process of coal and rock mass. However, the expansion of crystal volume will result in inhomogeneous deformation lattice creating a dislocation. Fig. 4 shows the slip volume expansion diagram. Fig. 5 indicates schematic diagram of the dislocation deformation.

Symmetrical processing is in accordance with the lattice structure, and we take $1 / 4$ as the research object. Assume that crystal deformation process in the lattice moves parallelly. The lattice average size is recorded by $D$. And the chemical potential of the crystal on the interface is:

$\left\{\begin{array}{l}\mu_{x}=\mu_{0}+e q^{*} \phi(x, t)+\Omega \sigma_{x} \\ \mu_{y}=\mu_{0}+e q^{*} \phi(y, t)+\Omega \sigma_{y}\end{array}\right.$

where, $e$ is the electron charge. $q^{*}$ is the effective charge number. $\phi$ is the electric potential. $\Omega$ represents the atomic volume. $\sigma_{x}$ and $\sigma_{y}$ are the normal stress on the crystal at the interface of $x$ and $y$ directions, respectively. Among, positive represents tensile stress, and negative represents pressure stress.

The diffusion flux along the crystal interface as:

$\left\{\begin{array}{l}J_{x}=\frac{\delta_{b} S_{b}}{k T}\left(e q^{*} \frac{\partial \phi}{D \partial x}+\Omega \frac{\partial \sigma_{x}}{D \partial x}\right) \\ J_{y}=\frac{\delta_{b} S_{b}}{k T}\left(e q^{*} \frac{\partial \phi}{\partial \partial y}+\Omega \frac{\partial \sigma_{y}}{D \partial y}\right)\end{array}\right.$

where, $S_{b}$ is the diffusion coefficient of the crystal interface. $\delta_{b}$ is the thickness of the crystal interface. $k$ is the Boltzmann constant. $T$ is the absolute temperature.

According to the condition of conservation of mass:

$\frac{\partial J_{x}}{D \partial x}+\nu_{x}=0$
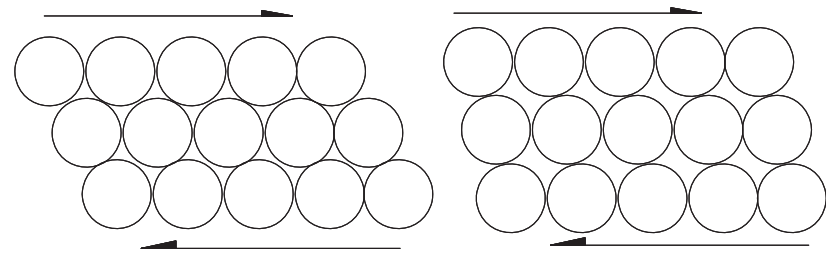

Fig. 4. Volume expansion produced by slip.

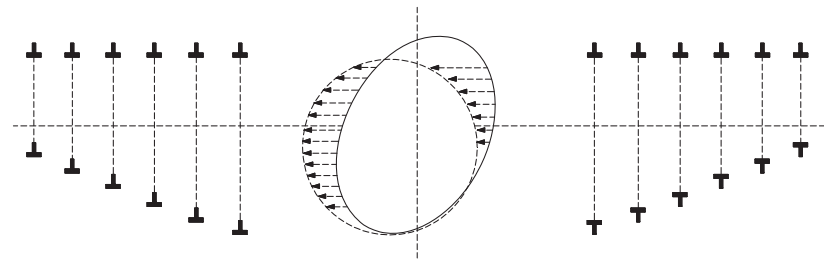

Fig. 5. Dislocation caused by deformation. 
$\frac{\partial J_{y}}{D \partial y}+\nu_{y}=0$

where, $\nu_{x}$ and $\nu_{y}$ are the rate of quality accumulation or consumption of the crystal on the interface, respectively. Positive represents the accumulation. On the contrary, negative represents the consumption.

Into the formula (20):

$\left\{\begin{array}{l}\frac{\Omega \delta_{b} S_{b} \partial^{2} \sigma_{X}}{k T D^{2} \partial \chi^{2}}+\nu_{x}=0 \\ \frac{\Omega \delta_{b} S_{b} \partial^{2} \sigma_{y}}{k T D^{2} \partial y^{2}}+\nu_{y}=0\end{array}\right.$

Assuming $\rho_{0}$ to be the reference value of charge density, and $\eta$ the resistivity. The one-dimensional treatment is:

$\bar{\rho}=\rho / \rho_{0}$

$\bar{\sigma}=\frac{\Omega \sigma}{e q^{*} \eta \rho_{0} D}$

$\bar{t}=\frac{\delta_{b} S_{b} e q^{*} \eta \rho_{0}}{k T D^{2}} t$

$\bar{\nu}=\frac{k T D^{2}}{\delta_{b} S_{b} e q^{*} \eta \rho_{0}} \nu$

Eq. (23) can be transformed into:

$\left\{\begin{array}{l}\frac{\partial^{2} \bar{\sigma}_{x}}{\partial x^{2}}+\bar{\nu}_{x}=0 \\ \frac{\partial^{2} \bar{\sigma}_{y}}{\partial y^{2}}+\bar{\nu}_{y}=0\end{array}\right.$

On the type points can be:

$\left\{\begin{array}{l}\bar{\sigma}_{x}=-\frac{1}{2} \bar{\nu}_{x}+C_{1} x+C_{2} \\ \bar{\sigma}_{y}=-\frac{1}{2} \bar{\nu}_{y}+C_{3} y+C_{4}\end{array}\right.$

where, $\bar{\nu}_{x}, \bar{\nu}_{y}, C_{1}, C_{2}, C_{3}$ and $C_{4}$ all can be determined through the boundary conditions.

Assuming that the average strain rate is $\varepsilon$ in the process of the lattice deformation. Crystal interface charge generated in the expansion process of lattice slip and inhomogeneous deformation can be expressed as:

$q^{*}=\frac{\varepsilon \bar{t} k T D^{2}}{\delta_{b} S_{b} e \eta \rho}$

\section{Experimental study of the piezoelectric effect of coal and rock}

\subsection{Coal samples and devices}

The coal sample size is $50 \mathrm{~mm} \times 50 \mathrm{~mm} \times 100 \mathrm{~mm}$. The experiment instruments and equipments consist primarily of loading system, signal data acquisition systems, data recording system and electromagnetic shielding systems. The charge signal generated by the deformation and fracture of coal or rock is weak. So, the weak charge signal is converted to a voltage signal $[19,20]$ by charge sensor. Fig. 6 shows the piezoelectric experimental setup of coal and rock.

\subsection{Analysis of piezoelectric effect of coal and rock}

Fig. 7 indicates the results of the coal sample damage in piezoelectric experiment. Cracks develop through vertical direction, transverse crack expand continuously, consequently, the crack breaks $[19,20]$ at the end of coal sample. By interface barrier

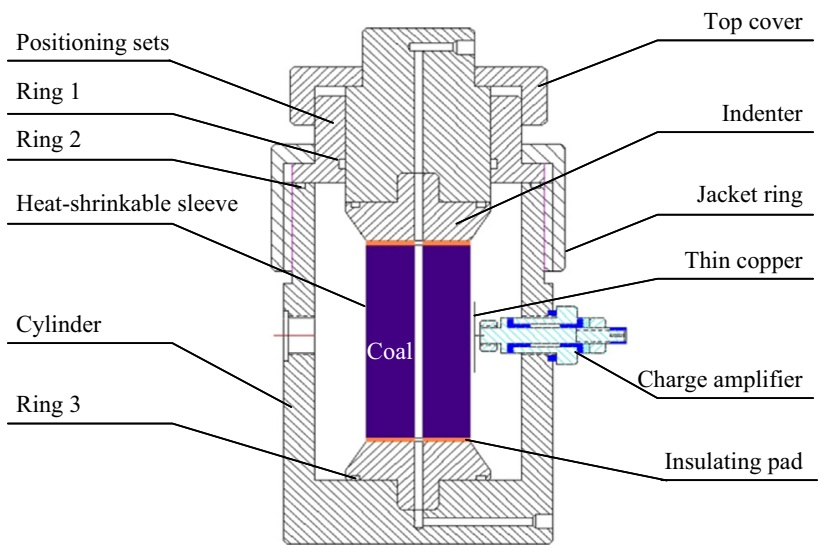

Fig. 6. Piezoelectric experimental setup of coal and rock.

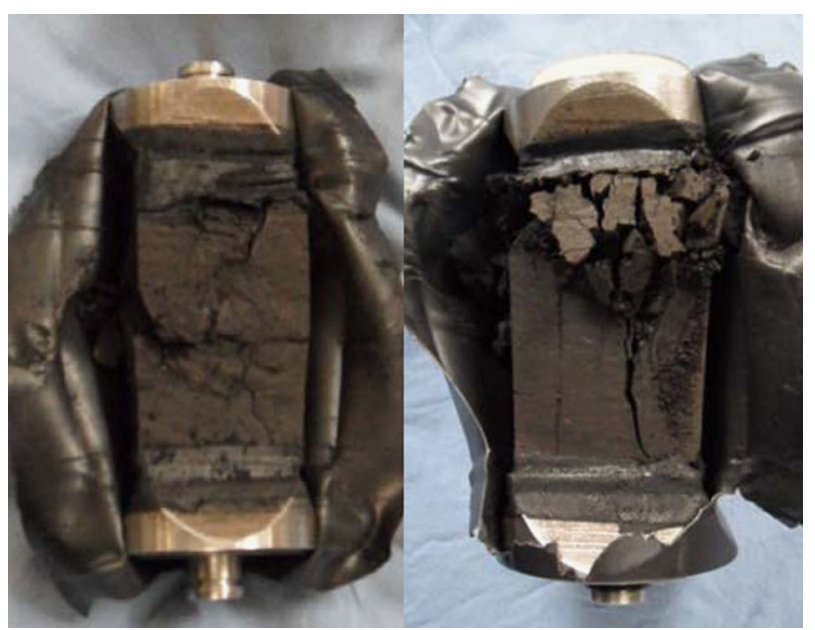

Fig. 7. The destruction of coal sample.

formation mechanism of micro-crack and theoretical model of crack sliding friction electricity, it is known that the potential difference (potential) caused by the interfacial charge transfer and barrier increasing in the process of coal sample crack propagation. Therefore, the potential changes measured by piezoelectric experiments can reflect the law of the charge generated in the process of coal rupture.

A set of data result is analyzed for the experimental results are consistent. Fig. 8 indicates the relations between stress and monitoring potential changes with time. The piezoelectric experimental results show that the signal is weak. The reason is that the original crack close and potential value is very small at the initial. With the constant load, the newborn crack interface barrier increase causing signal and potential value enhance. Before the coal sample rupture, the sliding friction of crack interface is the most intense, and the amplitude of monitor potential changes is also the most obvious. The crack interface friction decreases suddenly after coal sample rupture. And then, the charge transfer $[21,22]$ speed up instantly, and the interface barrier also increase instantly. In addition, the charge transfer is significantly accelerated before the coal sample rupture. That is, the crack interface barrier increase rapidly before the rupture of coal sample. Therefore, the precursor information can be obtained before the coal sample breakdown. The monitoring results can predict the extent and state of coal or rock breakage. 


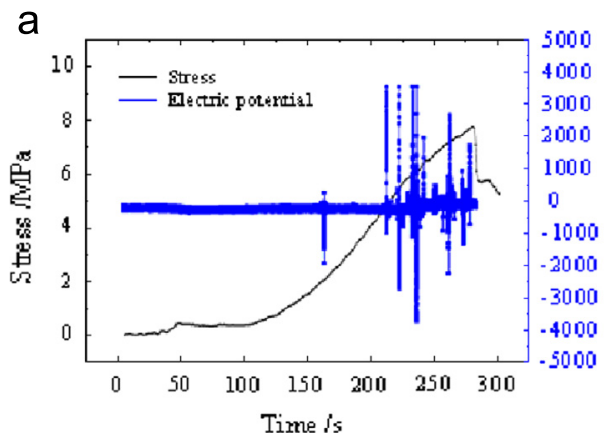

b

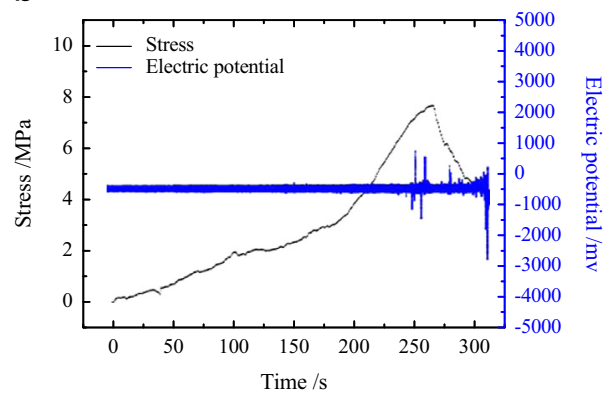

C

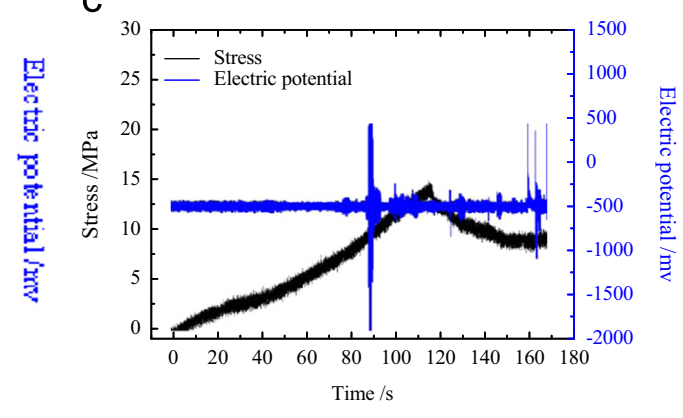

d

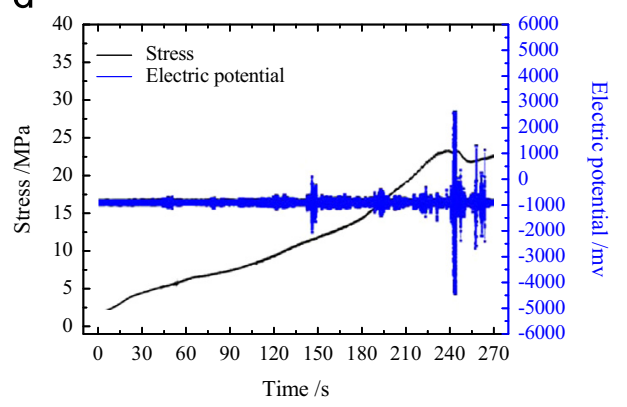

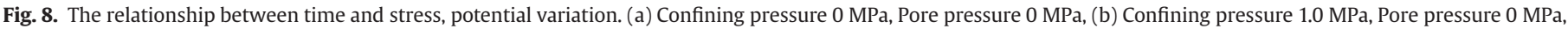
(c) Confining pressure 1.0 MPa, Pore pressure 0.5 MPa and (d) Confining pressure 3.0 MPa, Porepressure $0.8 \mathrm{MPa}$.

\section{Conclusions}

Based on the study of formation of micro-crack interface barrier and piezoelectric effect of coal and rock mass, the following conclusions can be drawn:

(1) The micro-cracks of coal or rock expand constantly under external loads. The slip dislocations and inhomogeneous deformation of crack interface and lattice cause charge breakthrough. At the same time, barriers form on the crystal interface.

(2) The charge generated in the friction process of micro-crack slip dislocation and deformation is theoretically analyzed. Then, a theoretical model of micro-crack slip friction charge is established.

(3) The crack interface barrier and the potential value increase constantly in the compression process of coal sample. Also before the coal sample rupture, sliding friction between crack interfaces is the strongest, the barrier is the highest. Moreover, the transfer speed of charge accelerated, and potential amplitude change is most obvious.

(4) Both the crack interface barrier and the potential amplitude get a major change before the coal sample fracture. The precursor information can be obtained before the coal sample breakdown. The monitoring results provide the basis for the prediction of the extent and status of coal or rock break.

\section{Acknowledgments}

This work was financially supported by National Natural Science Foundation No. 11172121, 51004061 and National Basic Research Program of China under Grant No.2010CB226803.

\section{References}

[1] Cahn RW. Physical metallurgy. London: North-Holland; 1970.
[2] Hirsch PB. The physics of metals detects.London: Cambridge University Press; 1975.

[3] Honeycombe RWK. The plastic deformation of metals.London: Edward Arnold; 1968.

[4] Hirth JP, Lothe J. Theory of dislocations.New York: McGraw-Hill; 1968.

[5] Brady BT, Rowell GA. Laboratory investigation of the electrodynamics of rock fracture. Nature 1986;321:488-92.

[6] Xueqiu HE, Baisheng NIE, Jun HE, et al. Study on electromagnetic emission characteristics in roof failure. Chinese Journal of Rock Mechanics and Engineering 2007;26:2935-40.

[7] Wu XP, Shio XJ, Guo ZQ. Study on the electrification of granite samples under compression. Chinese Journal of Geophysics 1990;33:208-11.

[8] Enomoto Y, Shimamoto T, Tsutumi A, et al. Rapid electric charge fluctuation prior to rock fracturing: its potential use for an immediate earthquake precursor. In: Proceedings of international workshop on electromagnetic phenomena related to earthquake prediction. Tokyo; 6-8 September 1993. p. 64-65.

[9] Takeuchi A, Bobby WSL, Friedemann TF. Current and surface potential induced by stress activated positive holes in igneous rocks. Physics and Chemistry of the Earth 2006;31:240-7.

[10] Kuksenko VS, Inzhevatkin IE, Manzhikov BT, et al. Physical and methodological principles of rock burst prediction. Soviet Mining Science 1988;24:6-17.

[11] Ivanov VV, Egorov PV, Pimonov AG. Statistical theory of emission process in loaded structurally in homogeneous rocks and the problem of predicting dynamic phenomena. Soviet Mining Science 1991;26:343-9.

[12] Niston U. Electromagnetic emission accompanying fracture of quartz-bearing rocks. Geophysical Research Letters 1977;4:333-6.

[13] Volarovich MP, Parkhomenko EI. The piezoelectric effect of rock. An SSSR, ser Geofiz 1955:2:215-22.

[14] Tan HL. Combined macroscopic and microscopic investigation on the fracture process of materials. PhD Thesis. Tsinghua University, Beijing; 1996.

[15] Huang K. Solid state physics.Beijing: Peking University Press; 2009.

[16] Epifanov EJ. Solid state physics.Moscow: Mir Publisher; 1979.

[17] Yang SL, Xu TD, Li QF. Physics of materials. Haerbin:Harbin Institute of Technology Press; 2004.

[18] Shao SS. Analysis of diffusion, stress and deformation of microstructures applied by loading of mechanical and electro-mechanical coupling. PhD Thesis. East China University of Science and Technology, Guangzhou; 2011.

[19] Li ZH, Wang EY, Liu ZT, et al. Study surface on characteristics and rules of potential during coal fracture. Journal of China University of Mining and Technology 2009;38:187-92.

[20] Wang EY, He XQ Dou LM, et al. Electromagnetic radiation characteristics of coal and rocks during excavation in coal mine and their application. Chinese Journal of Geophysics 2005;48:216-21.

[21] Ogawa T, Oike K, Miura T. Electromagnetic radiation from rocks. Journal of Geophysical Research 1985;90:6245-9.

[22] He XQ, Nie BS, Wang EY. Electromagnetic emission forecasting technology of coal or rock dynamic disasters in mine. Journal of China Coal Society 2007;32:56-9. 